\title{
DISTRIBUTIONAL DERIVATION OF AN ASYMPTOTIC EXPANSION ${ }^{1}$
}

\author{
R. WONG
}

\begin{abstract}
An alternative derivation of the asymptotic expansion of multiple Fourier transforms is presented. The present approach is based on the use of distributions. With some modifications, this method can also be applied to other integral transforms with oscillatory kernels such as the Hankel transform.
\end{abstract}

1. Introduction. Recently, a new method based on the use of distributions has been introduced into the asymptotic evaluation of integrals; see [1], [3], [4], [11]. An advantage of this approach is that it leads to a particularly illuminating construction of error terms associated with the asymptotic expansions. However, this method has been applied, so far, only to integrals whose kernels are algebraic functions, for instance, the Stieltjes transform [3], the Riemann-Liouville fractional integral [4], and a Mellin convolution of two algebraically dominated functions [11, §11]. Although a different method has been devised recently for the same purpose for integrals with oscillatory kernels, such as the one-sided Fourier integral [5], the Hankel transform [10], and the multidimensional Fourier transform [9], it is nevertheless of interest to know whether the distributional approach can be applied with equal advantage to the second-mentioned class of integrals. It turns out that this is indeed the case, and the purpose of the present note is simply to show this. Since the arguments for the various cases involving oscillatory integrands are similar, we shall illustrate the technique by only giving an alternative derivation of the asymptotic expansion of the multidimensional Fourier transform [9]:

$$
\hat{f}(t)=\frac{1}{(2 \pi)^{n / 2}} \int_{R^{n}} f(x) e^{i t \cdot x} d x
$$

where $x=\left(x_{1}, \ldots, x_{n}\right), t=\left(t_{1}, \ldots, t_{n}\right), t \cdot x=t_{1} x_{1}+\cdots+t_{n} x_{n}$ and $f$ is a complex-valued locally integrable function on $R^{n}$. In (1) it is only assumed that the Cauchy limit $\lim _{\nu \rightarrow \infty} \int_{|x|<\nu} f(x) e^{i t \cdot x} d x$ exists for every $t \in R^{n}$, where $|x|=\sqrt{x_{1}^{2}+\cdots+x_{n}^{2}}$. Thus the function $f$ need not belong to $L^{1}\left(R^{n}\right)$.

2. Notation and assumptions. The notation to be used in this note will be similar to that used in either [9] or [6]. If $p=\left(p_{1}, \ldots, p_{n}\right)$ is a multi-index of nonnegative integers then we put $|p|=p_{1}+\cdots+p_{n}, x^{p}=x_{1}^{p_{1}} \ldots x_{n}^{p_{n}}, D^{p}=D_{1}^{p_{1}} \ldots D_{n}^{p_{n}}$,

Received by the editors November 27, 1979.

AMS (MOS) subject classifications (1970). Primary 41A60; Secondary 42A92, 46F10.

Key words and phrases. Asymptotic expansions, distribution, Fourier transform.

${ }^{1}$ This research was partially supported by the National Science and Engineering Research Council of Canada under Contract A7359. 
where $D_{j}=\partial / \partial x_{j}$, and

$$
D_{p}=(i)^{-|p|} D^{p}=\left(\frac{1}{i} \frac{\partial}{\partial x_{1}}\right)^{p_{1}} \cdots\left(\frac{1}{i} \frac{\partial}{\partial x_{n}}\right)^{p_{n}} .
$$

For any open set $\Omega \subset R^{n}$ and for each nonnegative integer $m$, the set $C^{m}(\Omega)$ consists of all complex functions $f$ in $\Omega$ whose derivative $D^{p} f$ exists and is a continuous function in $\Omega$ for each multi-index $p$ with $|p|<m$. The Laplacian is denoted by $\Delta=\partial^{2} / \partial x_{1}^{2}+\cdots+\partial^{2} / \partial x_{n}^{2}$.

The following assumptions are exactly those adopted in [9].

$\left(\mathrm{A}_{1}\right) f \in C^{2 m}\left(R^{n} \backslash\{0\}\right), m$ being a nonnegative integer. $f(x)$ is expressible in the form

$$
f(x)=\sum c_{p q} x^{p} r^{q}+\varphi(x)
$$

where the $q$ 's are real numbers, the $p$ 's are multi-indices, $r=\sqrt{x_{1}^{2}+\cdots+x_{n}^{2}}$ and the sum is finite. For all $p$ and $q$ under the summation, we require $q+|p|>-n$, and if $Q=\max \{q+|p|\}$ then $2 m-1<Q+n<2 m+1$.

$\left(\mathrm{A}_{2}\right)$ As $r \rightarrow 0^{+}$, the remainder $\varphi(x)$ in (2) satisfies

$$
\left(\Delta^{j} \varphi\right)(x)=O\left(r^{Q-2 j+1}\right), \quad j=0,1, \ldots, m,
$$

if $Q+n \neq 2 m-1$, and

$$
\left(\Delta^{j} \varphi\right)(x)=O\left(r^{Q-2 j+2}\right), \quad j=0,1, \ldots, m,
$$

if $Q+n=2 m-1$.

$\left(A_{3}\right)$ For some $\rho>0$, each of the integrals

$$
\int_{|x|>\rho}\left(\Delta^{j} f\right)(x) e^{i t \cdot x} d x, \quad j=0,1, \ldots, m,
$$

converges uniformly for all sufficiently large $|t|$.

REMARK. Under the above assumptions, the Fourier transform $\hat{f}(t)$, as given in (1), and the Fourier transform

$$
\left(\Delta^{m} \varphi\right)^{\wedge}(t)=\frac{1}{(2 \pi)^{n / 2}} \int_{R^{n}}\left(\Delta^{m} \varphi\right)(x) e^{i t \cdot x} d x i
$$

both exist uniformly for all sufficiently large values of $|t|$; see [9, Remark (iii)]. From this it follows that $\hat{f}(t)$ and $\left(\Delta^{m} \varphi\right)^{\wedge}(t)$ are continuous functions of $t \in R^{n}$. Also, it is easy to see that for each $j=0,1, \ldots, m,\left(\Delta^{j} \varphi\right)(x)$ is locally integrable on $R^{n}$ and of at most finite algebraic growth at infinity.

3. Distributions. Let $\mathcal{S}$ denote the space of rapidly decreasing functions and let $\mathcal{S}^{\prime}$ be the space of tempered distributions [6, pp. 168-174]. If $\eta \in \mathcal{S}$ and $\Lambda \in \mathcal{S}^{\prime}$ then we write $\langle\Lambda, \eta\rangle$ for the action of $\Lambda$ on $\eta$.

It is well known that any locally integrable function $f(x)$ on $R^{n}$ of finite algebraic growth at infinity generates a distribution $f \in \mathcal{S}^{\prime}$ by means of the definition

$$
\langle f, \eta\rangle=\int_{R^{n}} f(x) \eta(x) d x
$$


see, for example, [6, p. 175]. Thus, in particular, the distribution associated with the function $r^{\lambda}, \operatorname{Re} \lambda>-n$, is defined by

$$
\left\langle r^{\lambda}, \eta\right\rangle=\int_{R^{n}} r^{\lambda} \eta(x) d x
$$

see $[2$, p. 71].

If $p$ is a multi-index and $u \in \mathcal{S}^{\prime}$ then the formula

$$
\left\langle D^{p} u, \eta\right\rangle=(-1)^{|p|}\left\langle u, D^{p} \eta\right\rangle
$$

defines a distribution $D^{p} u \in \mathcal{S}^{\prime}$; see [6, §6.12]. If $f$ and $D^{p} f$ are both locally integrable in $R^{n}$ with at most finite algebraic growth at infinity then, by integration by parts, one can show that

$$
\int_{R^{n}}\left(D^{p} f\right)(x) \eta(x) d x=(-1)^{|p|} \int_{R^{n}} f(x)\left(D^{p} \eta\right)(x) d x
$$

for every $\eta \in \mathcal{S}$, under the hypotheses that $f$ has continuous partial derivatives of all order up to $|p|-1$, all these derivatives have at most finite algebraic growth at infinity, and all partial derivatives of order $|p|$ are locally absolutely continuous in each variable $x_{1}, \ldots, x_{n}$. Note that these conditions are weaker than those given in [6, p. 144]. A rigorous proof of (7) can be provided easily by using the argument given in [8, p. 80] and a remark made in [2, p. 21, $\$ 2.2$, paragraph 2]. The validity of equation (7) shows that the distributional derivative and the ordinary derivative are equal (in the sense of distributions).

What we need is a variant of (7), namely

$$
\int_{R^{n}} f(x)(\Delta \eta)(x) d x=\int_{R^{n}}(\Delta f)(x) \eta(x) d x
$$

for every $\eta \in \mathcal{S}$. This will imply that the distributional and the ordinary Laplacians are equal also. For equation (8) to hold we require that $f$ has continuous second partial derivatives in $R^{n}$ except possibly at the origin, $f$ grows at most algebraically at infinity, and $f(x)=O\left(r^{\alpha}\right)$, as $r \rightarrow 0^{+}$, with $\alpha+n>0$. The result (8) follows easily from an application of Green's theorem [8, p. 88]. To be more rigorous, Green's theorem should be applied to the shell domain $S=\left\{x \in R^{n}\right.$; $\varepsilon \leqslant|x| \leqslant \rho\}$. Relation (8) then results as a limit when $\varepsilon \rightarrow 0$ and $\rho \rightarrow+\infty$.

4. Fourier transforms. If $\eta \in \mathcal{S}$ then $\hat{\eta}$, as defined in (1), exists and belongs to $\delta$; see [6, p. 168]. If $u \in \mathcal{S}^{\prime}$ then the Fourier transform $\hat{u}$ is defined by the equation

$$
\langle\hat{u}, \eta\rangle=\langle u, \hat{\eta}\rangle, \quad \eta \in \mathcal{S} .
$$

It is well known that $\hat{u} \in \mathcal{S}^{\prime}$ and the following identities [6, p. 176] hold: if $P$ is a polynomial in $n$ variables, say, $P(x)=\Sigma c_{p} x^{P}$, and if the differential operator $P(D)$ is defined by $P(D)=\Sigma c_{p} D_{p}$, then

$$
(P(D) u)^{\wedge}=P(-t) \hat{u} \text { and }(P u)^{\wedge}=P(D) \hat{u} .
$$

If $f$ is locally integrable in $R^{n}$ and increases no more rapidly than an algebraic function, and if the Cauchy limit in the integral of (1) exists uniformly in $t \in R^{n}$, then the distributional Fourier transform of $f$ given in (9) and the ordinary Fourier 
transform of $f$ given in (1) are equal as functionals; see [2, p. 200]. The following result is crucial to our main theorem in $\$ 5$.

Lemma. Let $|p|+q+n>0$. If $q+n \neq-2 l, l=0,1,2, \ldots$, then

$$
\left(x^{p} r^{q}\right)^{\wedge}=L(q) D_{p}\left(|t|^{-q-n}\right) \text { in } \delta^{\prime} \text {, }
$$

where $L(q)=2^{q+n / 2} \Gamma[(q+n) / 2] / \Gamma(-q / 2)$. If $q+n=-2 l, l=0,1,2, \ldots$, then

$$
\left(x^{p} r^{q}\right)^{\wedge}=L^{*}(q) D_{p}\left(|t|^{2 l} \log |t|\right) \text { in } \mathcal{S}^{\prime} \text {, }
$$

where $L^{*}(q)=(-1)^{l+1} / 2^{n / 2+2 l-1} l ! \Gamma\left(\frac{1}{2} n+l\right)$.

Note that the value of $L^{*}(q)$ given in [9] is slightly in error.

Proof. It is known that

$$
\left(r^{q}\right)^{\wedge}=L(q)|t|^{-q-n} \text { in } \mathcal{S}^{\prime}
$$

if $q+n \neq-2 l, l=0,1,2, \ldots$, and

$$
\left(r^{q}\right)^{\wedge}=L^{*}(q)|t|^{2 l} \log |t|+K|t|^{2 l} \text { in } \mathcal{S}^{\prime}
$$

if $q+n=-2 l, l=0,1,2, \ldots$, where $K$ is some constant; see [7, pp. 257-258] and [2, pp. 194-195]. Thus (11) follows immediately from the second equation in (10). Since $D_{p}\left(|t|^{2 l}\right)=0$ for $|p|>2 l$, (12) also follows immediately from the second equation in (10), thus proving the lemma.

5. Asymptotic expansions. We now return to equation (2). Note that each function in this equation generates a distribution in $\mathcal{S}^{\prime}$ by means of the definition (4), and that these distributions are again related via this equation, i.e.,

$$
f=\sum c_{p q} x^{p} r^{q}+\varphi \text { in } \mathcal{S}^{\prime} .
$$

Taking (distributional) Fourier transforms on both sides, we have by our lemma

$$
\hat{f}=\sum^{\prime} c_{p q} L(q) D_{p}\left(|t|^{-q-n}\right)+\sum^{\prime \prime} c_{p q} L^{*}(q) D_{p}\left(|t|^{2 l} \log |t|\right)+\hat{\varphi}
$$

in $\mathcal{S}^{\prime}$, where $\Sigma^{\prime}$ excludes those $q$ 's for which $q+n$ is a negative even integer, and $\Sigma^{\prime \prime}$ includes only those $q$ 's for which $q+n=-2 l$, a negative even integer. Applying the first equation in (10) $m$ times gives

$$
\left(\Delta^{m} \varphi\right)^{\wedge}=(-1)^{m}|t|^{2 m} \hat{\varphi} \quad \text { in } \mathcal{S}^{\prime} .
$$

Inserting (17) in (16), we have as functionals

$$
\begin{aligned}
|t|^{2 m} \hat{f}= & \sum^{\prime} c_{p q} L(q)|t|^{2 m} D_{p}\left(|t|^{-q-n}\right) \\
& +\sum^{\prime \prime} c_{p q} L^{*}(q)|t|^{2 m} D_{p}\left(|t|^{2 l} \log |t|\right)+(-1)^{m}\left(\Delta^{m} \varphi\right)^{\wedge} .
\end{aligned}
$$

In view of the remark in $\$ 2$ and the remark in $\$ 4$ concerning the ordinary and distributional Fourier transforms, we see that each distribution in (18) is defined by a locally integrable function in $R^{n}$, and hence that equation (18) holds not only as functionals but also pointwise almost everywhere in $R^{n}$. Since all functions involved are in fact continuous (see again the remark in \$2), equation (18) is valid for every $t \in R^{n}$.

Therefore, we have established the following theorem, which is the main result in [9]. 
THEOREM. Under the conditions $\left(\mathrm{A}_{1}\right),\left(\mathrm{A}_{2}\right)$ and $\left(\mathrm{A}_{3}\right)$, we have

$$
\begin{aligned}
\hat{f}(t)= & \sum^{\prime} c_{p q} L(q) D_{p}\left(|t|^{-q-n}\right) \\
& +\sum^{\prime \prime} c_{p q} L^{*}(q) D_{p}\left(|t|^{2 l} \log |t|\right)+\delta_{m}(t),
\end{aligned}
$$

where the sums $\Sigma^{\prime}$ and $\Sigma^{\prime \prime}$ are as given in (16) and the remainder satisfies

$$
\delta_{m}(t)=\left((-1)^{m} / \mid t^{2 m}\right)\left(\Delta^{m} \varphi\right)^{\wedge}(t) .
$$

REMARK. As already pointed out in $\S 2$, the Fourier transform $\left(\Delta^{m} \varphi\right)^{\wedge}(t)$ given in (3) exists uniformly for all sufficiently large values of $t \in R^{n}$. Hence, by a generalization of the Riemann-Lebesgue lemma, we have

$$
\delta_{m}(t)=o\left(|t|^{-2 m}\right) \text { as }|t| \rightarrow \infty .
$$

Thus (19) is indeed an asymptotic expansion.

\section{REFERENCES}

1. P. Durbin, Asymptotic expansion of Laplace transforms about the origin using generalized functions, J. Inst. Math. Appl. 23 (1979), 181-192.

2. I. M. Gel'fand and G. E. Shilov, Generalized functions, vol. 1, Academic Press, New York, 1964.

3. J. P. McClure and R. Wong, Explicit error terms for asymptotic expansions of Stieltjes transforms, J. Inst. Math. Appl. 22 (1978), 129-145.

4. , Exact remainders for asymptotic expansions of fractional integrals, J. Inst. Math. Appl. 24 (1979), 139-148.

5. F. W. J. Olver, Error bounds for stationary phase approximations, SIAM J. Math. Anal. 5 (1974), 19-29.

6. W. Rudin, Functional analysis, McGraw-Hill, New York, 1973.

7. L. Schwartz, Théorie des distributions, Hermann, Paris, 1966.

8. __ Mathematics for physical sciences, Addison-Wesley, Reading, Mass., 1966.

9. P. N. Shivakumar and R. Wong, Asymptotic expansion of multiple Fourier transforms, SIAM J. Math. Anal. 10 (1979), 1095-1104.

10. R. Wong, Error bounds for asymptotic expansions of Hankel transforms, SIAM J. Math. Anal. 7 (1976), 799-808.

11. Error bounds for asymptotic expansions of integrals, SIAM Rev. (to appear).

Departiment of Mathematics, University of Manitoba, Winnipeg, Mantroba, Canada R3T 2N2 